\title{
Planar tetracoordinate carbons in allene-type structures
}

Received 00th January 20xx,

Meng-hui Wang, ${ }^{\mathrm{a}}$ Mesías Orozco-Ic, ${ }^{\mathrm{b}}$ Luis Leyva-Parra, ${ }^{\mathrm{c}}$ William Tiznado, ${ }^{\mathrm{c}}$ Jorge Barroso, ${ }^{* \mathrm{~b}}$ Yi-hong Ding, ${ }^{* d}$ Zhong-hua Cui*ae and Gabriel Merino. ${ }^{* c}$

Accepted 00th January 20xx

DOI: $10.1039 / \times 0 \times x 00000 x$

The exhaustive exploration of the potential energy surfaces of $\mathrm{CE}_{2} \mathrm{M}_{2}(\mathrm{E}=\mathrm{Si}-\mathrm{Pb}, \mathrm{M}=\mathrm{Li}$, and $\mathrm{Na})$ revealed seven global minima containing a planar tetracoordinate carbon (ptC). The design was based on a $\pi$-localization strategy, resulting in a ptC embedded in a linear or a bent allene-type $E=C=E$ motif. The magnetic response showed a $\sigma$-aromaticity in the bent $E=C=E$ fragments. The bonding analysis indicated that the ptCs form C-E covalent bonds and C-M electrostatic interactions.

In 1874, van't Hoff and Lebel independently established one of the cornerstones of organic chemistry by stating that a carbon atom with four substituents has a tetrahedral arrangement. ${ }^{1,2}$ For almost a century, the tetracoordinate carbon structures were rationalized according to this premise, and then gradually, carbon revealed an architecture versatility beyond these widely accepted rules. The umbrella configuration, ${ }^{3,4}$ in which one of the tetrahedral bonds is upside down; the pyramidal arrangement with the carbon atom at the top and its four ligands at the base; $;^{5-7}$ the bisphenoidal or half-planar topology found in carbides and organometallic clusters; ${ }^{8-10}$ and the planar tetracoordinate carbon (ptC), are some exemplars. ${ }^{11-20}$ Molecules with these bizarre topologies have been synthesized or experimentally detected, expanding our understanding of tetracoordinate carbon species.

Particularly intriguing are ptCs, whose design strategy was initially proposed more than fifty years ago by Hoffmann, Alder, and Wilcox ${ }^{11}$ based on analysing the molecular orbitals of planar methane as a response to the hypothetical transition state

\footnotetext{
a. Institute of Atomic and Molecular Physics, Key Laboratory of Physics and Technology for Advanced Batteries (Ministry of Education), Jilin University, Changchun 130023, China. E-mail: zcui@jlu.edu.cn

b. Departamento de Física Aplicada, Centro de Investigación y de Estudios Avanzados, Unidad Mérida, km. 6 Antigua carr. a Progreso. Apdo Postal 73, Cordemex, Mérida, Yuc., México. E-mail:gmerino@cinvestav.mx, jorge.barroso@cinvestav.mx

c. Computational and Theoretical Chemistry Group, Departmento de Ciencias Químicas, Facultad de Ciencias Exactas, Universidad Andres Bello, República 498, Santiago, Chile

d. Key Laboratory of Carbon Materials of Zhejiang Province, College of Chemistry and Materials Engineering, Wenzhou University, Wenzhou 325035, P. R. China. Email:yhdd@jlu.edu.cn

e. Beijing National Laboratory for Molecular Sciences, China

Electronic Supplementary Information (ESI) available: [details of any supplementary information available should be included here]. See DOI: 10.1039/x0xx00000x
}

suggested by Monkhorst two years earlier. ${ }^{12}$ Essentially, the strategy consists of using $\sigma$-donor/ $\pi$-acceptor ligands or delocalizing the carbon's lone pair into the system framework (see the left side of Fig. 1). A vast number of molecules with a ptC have been designed using such an approach. ${ }^{21-33}$ Quite recently, we suggested an alternative to stabilize a ptC compound by localizing $\pi$-orbitals between the central carbon and a ligand in the $\mathrm{CEM}_{3}$ compounds. The formation of a double $\mathrm{C}=\mathrm{E}$ bond can compensate for the weakening of $\mathrm{C}-\mathrm{M}$ and ligandligand interactions (see right side of Fig. 1). ${ }^{34}$ This strategy has been successfully applied for designing global minima structures with a ptC like $\mathrm{CAl}_{3} \mathrm{E}(\mathrm{E}=\mathrm{P}, \mathrm{As}, \mathrm{Sb}$, and $\mathrm{Bi}),{ }^{35} \mathrm{CLi}_{3} \mathrm{E}(\mathrm{E}$ $=\mathrm{As}, \mathrm{Sb}$, and $\mathrm{Bi}), \mathrm{CLi}_{3} \mathrm{E}^{+}\left(\mathrm{E}=\right.$ Group 16 element), ${ }^{36}$ and $\mathrm{C}_{2} \mathrm{Al}_{4} \cdot{ }^{37}$ Also, it was recently exploiting for predicting planar pentacoordinate silicon and germanium atoms. ${ }^{38}$ This reflects the efficiency of the $\pi$-localization strategy for designing planar hypercoordinate carbon atoms.

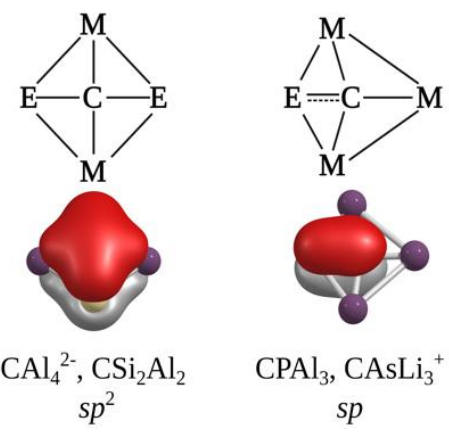

Fig. 1. The delocalized $\pi$-orbital for representative systems with $s p^{2}$ hybridization and the $\pi$-localized orbital in $s p$-systems. 
Table 1. Structure and relative energy $(\mathrm{kcal} / \mathrm{mol})$ of the lower-lying energy isomers of $\mathrm{CE}_{2} \mathrm{Li}_{2}(\mathrm{E}=\mathrm{Si}-\mathrm{Pb})$ at the $\mathrm{CCSD}(\mathrm{T}) /$ def2-TZVP level. Values in parentheses correspond to clusters with $\mathrm{Na}$.

\begin{tabular}{|c|c|c|c|c|c|}
\hline & $\begin{array}{c}\mathbf{A} \\
D_{2 h},{ }^{1} \mathrm{~A}_{\mathrm{g}}\end{array}$ & $\begin{array}{c}\text { B } \\
C_{2 v},{ }^{1} \mathrm{~A}_{1}\end{array}$ & $C_{s},{ }^{1} A^{\prime}$ & $C_{2 v},{ }^{3} \mathrm{~A}_{1}$ & $C_{2 v},{ }^{1} \mathrm{~A}_{1}$ \\
\hline $\mathrm{CSi}_{2} \mathrm{M}_{2}$ & $0.0(0.0)$ & $1.3(6.1)$ & $9.4(5.9)$ & $9.4(3.8)$ & $12.3 *(7.0)$ \\
\hline $\mathrm{CGe}_{2} \mathrm{M}_{2}$ & $0.8(0.0)$ & $0.0(4.7)$ & $7.6(3.5)$ & $9.5(4.4)$ & $10.6 *(4.8)$ \\
\hline $\mathrm{CSn}_{2} \mathrm{M}_{2}$ & $2.5(0.0)$ & $0.0(3.0)$ & $9.2(2.0)$ & $10.5(3.3)$ & $10.5 *(2.0)$ \\
\hline $\mathrm{CPb}_{2} \mathrm{M}_{2}$ & $5.0(0.9)$ & $0.0(1.2)$ & $10.1(-)$ & $12.5(4.1)$ & $11.0 *(0.0)$ \\
\hline
\end{tabular}

* means an imaginary frequency, and - for not available.

Herein, we extended this approach by incorporating a second $\mathrm{C}-\mathrm{E}$ double bond. The candidate series is $\mathrm{CE}_{2} \mathrm{M}_{2}(\mathrm{E}=\mathrm{Si}-$ $\mathrm{Pb}, \mathrm{M}=\mathrm{Li}$, and $\mathrm{Na}$ ). The global minima of seven of the eight possible combinations emerged as a structure with a ptC. The ptC may be embedded in a linear or a bent allene-type $E=C=E$ motif. According to the magnetic response, the bent allene-type $\mathrm{E}=\mathrm{C}=\mathrm{E}$ fragment presents $\sigma$-aromaticity, which plays an important role in stabilizing the cluster. The chemical bonding analysis revealed a mostly covalent C-E and an electrostatic C$\mathrm{M}$ nature for these ptCs. ${ }^{39}$

An extensive search of the potential energy surface of the $\mathrm{CE}_{2} \mathrm{M}_{2}(\mathrm{E}=\mathrm{Si}-\mathrm{Pb} ; \mathrm{M}=\mathrm{Li} / \mathrm{Na})$ series in the singlet and triplet state were performed (see the ESI). Table 1 shows the five recurrent lower-lying energy motifs. Note that all the global minima of the lithium-containing clusters correspond to a structure with a ptC. Furthermore, their corresponding second-lying isomer is also a ptC. The global minimum of $\mathrm{CSi}_{2} \mathrm{Li}_{2}$ is the only one adopting a $D_{2 h}$ symmetry (type-A) $1.3 \mathrm{kcal} / \mathrm{mol}$ lower in energy than the trapezoidal $C_{2 v}$ form (type-B), which is the most energetically favorable structure for the remaining ptCs with lithium. In contrast, within the sodium series, the trapezoidal arrangement (B) is the second least favorable isomer. Three of the four sodium clusters have a global minimum with a ptC belonging to $D_{2 h}$ symmetry. The exception is $\mathrm{CPb}_{2} \mathrm{Na}_{2}$, which collapses into a tricoordinate carbon about $1.0 \mathrm{kcal} / \mathrm{mol}$ lower in energy than the ptC species. Essentially, the lightest atoms provide a more suitable cavity to stabilize a ptC, i.e., using lithium on the one hand and silicon or germanium on the other guarantees a ptC as the most viable structure. A more extensive list of low-lying energy isomers is given in Fig. S1-S8.

According to Pyykkö covalent radii, ${ }^{40}$ the distance of a single C-Li bond is $2.08 \AA$, and a C-Na is $2.30 \AA$. Similarly, the double bond distances of $\mathrm{C}-\mathrm{Si}, \mathrm{C}-\mathrm{Ge}, \mathrm{C}-\mathrm{Sn}$, and $\mathrm{C}-\mathrm{Pb}$ are 1.91, 1.96, 2.15, and $2.19 \AA$, respectively. So, the C-M and C-E bond lengths, summarized in Table 2 and S1, suggest a single and a double bond, respectively. Indeed, for $\mathrm{CSi}_{2} \mathrm{Li}_{2}$ and $\mathrm{CGe}_{2} \mathrm{Li}_{2}$, the bond lengths are slightly shorter. However, the minimal $\mathrm{WBI}$ values (0.04 - 0.13) of the C-M contacts and charges imply that the interaction would be almost entirely electrostatic, and as expected, due to the $\pi$-location strategy, the WBI values for $C$ $E$ (1.24 - 1.50) point to a double C-E bond. The weakening of the interaction between the central atom and the alkali-metals are compensated by inducing multiple bonds with the elements of group 14 . In contrast, $\mathrm{CSi}_{2} \mathrm{Al}_{2}$, a prototype of an 18 valenceelectrons ptC stabilized via the conventional $\pi$-delocalization, has a similar C-Si distance ${ }^{13,14}$ but with a lower WBI value (1.02), indicating a single $\mathrm{C}-\mathrm{Si}$ bond. Moreover, the considerable WBI of C-Al (0.28) gives a hint of its $\pi$-delocalization.

The natural population analysis (NPA) indicates a charge on the ptC atom around $-1.91--2.00|\mathrm{e}|$ (Table 2 and S1), resulting from the charge transfer of $+1.90-+1.98|\mathrm{e}|$ from the alkalimetals and less than $+0.36|\mathrm{e}|$ from the atoms of group 14. Both charge distribution and $\mathrm{WBI}$ identify the $\mathrm{C}-\mathrm{M}$ and $\mathrm{C}-\mathrm{E}$ interactions to be mostly electrostatic and covalent, respectively. The electron density analysis corroborates the existence of a gradient path between the ptC and ligands. That is, the ptC atom is effectively coordinated with all ligands (Fig. S9). Moreover, the valence population of $\mathrm{CE}_{2} \mathrm{M}_{2}$ ( $\mathrm{E}=\mathrm{Si}-\mathrm{Pb}, \mathrm{M}=$ $\mathrm{Li}$, and $\mathrm{Na}$ ) presents a similar picture to the usual ptCs. That is, the $2 p_{x}$ and $2 p_{y}$ occupations are higher than the out-of-plane orbital $2 p_{z}\left(2 s^{1.48} 2 p_{x}{ }^{1.64} 2 p_{y}{ }^{1.45} 2 p_{z}{ }^{1.39}\right)$, revealing back-donation. Also, for type-A systems, the occupations of the $2 p_{y}$ orbital, which is in the direction of the lithium atoms, are significantly lower than in $2 p_{x}$ one, as a result of the $\mathrm{E}=\mathrm{C}=\mathrm{E}$ bond (vide infra). On the other hand, the remarkably lower $2 p_{z}$ population (1.11 $|\mathrm{e}|$ ) of the type-B $\mathrm{CSi}_{2} \mathrm{Li}_{2}$ implies a strong $\pi$-localization on the $\mathrm{E}=\mathrm{C}=\mathrm{E}$ fragment, consistent with the mentioned approach.

The picture emerged from the adaptive natural density partitioning (AdNDP) analysis confirms the bonding pattern described above. Both ptC types of $\mathrm{CSi}_{2} \mathrm{Li}_{2}$ (Fig. 2) and $\mathrm{CSi}_{2} \mathrm{Al}_{2}$ (Fig. S10) were used as examples of $\pi$-localization and $\pi$ delocalization bonding schemes, respectively. In both cases, there are two lone pairs on the $\mathrm{Si}$ atoms. For $\mathbf{A}$, eight valence electrons are delocalized in $3 c-2 e$ bonds, including two corresponding to the $\pi$-localization on $\mathrm{Si}-\mathrm{C}$-Si. The remaining electron pair is a $4 \mathrm{c}-2 \mathrm{e} \sigma$-bond. In the case of $\mathbf{B}$, there are two $5 c-2 e \quad \sigma$-bonds and three $3 c-2 e$, with one $\pi$-bond involving the $\mathrm{Si}-\mathrm{C}-\mathrm{Si}$ atoms. As expected, both ptC arrangements lack 5c-2e $\pi-$ bonds as in standard ptCs based on $\pi$-delocalization (Fig. S10). The charge decomposition analysis reveals that the lithium atoms play a significant role in stabilizing the $3 c-2 e$ in-plane $\pi$ bond (Fig. S11). 
Table 2. WBI, bond distances $(r, \AA \AA)$, natural charges $(q,|e|)$, and valence population of type-A and $\mathbf{B}$ for $\mathrm{CE}_{2} \mathrm{Li}_{2}(\mathrm{E}=\mathrm{Si}-\mathrm{Pb})$ at the $\operatorname{CCSD}(\mathrm{T}) / \mathrm{def} 2-\mathrm{TZVP}$ level.

\begin{tabular}{|c|c|c|c|c|c|c|c|c|c|c|c|c|}
\hline & A & & & & & & B & & & & & \\
\hline & $\mathrm{M}-\mathrm{C}$ & & $\mathrm{X}-\mathrm{C}$ & & & & $M-C$ & & $X-C$ & & & \\
\hline & WBI & $r$ & WBI & $r$ & $q(\mathrm{C})$ & Population & WBI & $r$ & WBI & $r$ & $q(\mathrm{C})$ & Population \\
\hline $\mathrm{CSi}_{2} \mathrm{Li}_{2}$ & 0.04 & 1.972 & 1.47 & 1.739 & -1.98 & $2 s^{1.48} 2 p_{x}{ }^{1.64} 2 p_{y}{ }^{1.45} 2 p_{z}{ }^{1.39}$ & 0.10 & 1.941 & 1.28 & 1.861 & -1.93 & $2 s^{1.61} 2 p_{x}{ }^{1.67} 2 p_{y}{ }^{1.53} 2 p_{z}{ }^{1.11}$ \\
\hline $\mathrm{CGe}_{2} \mathrm{Li}_{2}$ & 0.04 & 2.007 & 1.50 & 1.841 & -1.91 & $2 s^{1.52} 2 p_{x}{ }^{1.58} 2 p_{y}{ }^{1.44} 2 p_{z}{ }^{1.37}$ & 0.10 & 1.911 & 1.29 & 1.945 & -1.90 & $2 s^{1.65} 2 p_{x}{ }^{1.64} 2 p_{y}{ }^{1.52} 2 p_{z}{ }^{1.12}$ \\
\hline $\mathrm{CSn}_{2} \mathrm{Li}_{2}$ & 0.06 & 2.030 & 1.43 & 2.049 & -1.98 & $2 s^{1.63} 2 p_{x}^{1.55} 2 p_{y}^{1.44} 2 p_{z^{1.35}}$ & 0.12 & 1.918 & 1.24 & 2.157 & -2.00 & $2 s^{1.72} 2 p_{x}^{1.62} 2 p_{y}{ }^{1.52} 2 p_{z} p^{1.13}$ \\
\hline $\mathrm{CPb}_{2} \mathrm{Li}_{2}$ & 0.08 & 2.025 & 1.44 & 2.143 & -1.92 & $2 s^{1.67} 2 p_{x}{ }^{1.49} 2 p_{y}{ }^{1.43} 2 p_{z}{ }^{1.33}$ & 0.13 & 1.917 & 1.24 & 2.252 & -1.95 & $2 s^{1.74} 2 p_{x}{ }^{1.58} 2 p_{y}{ }^{1.50} 2 p_{z}{ }^{1.12}$ \\
\hline $\mathrm{CSi}_{2} \mathrm{Al}_{2}$ & 0.28 & 2.101 & 1.06 & 1.771 & -2.26 & $2 s^{1.50} 2 p_{x}{ }^{1.69} 2 p_{y}{ }^{1.67} 2 p_{z}{ }^{1.39}$ & 0.30 & 2.030 & 1.02 & 1.840 & -2.22 & $2 s^{1.56} 2 p_{x^{1.69}} p_{y^{1.66}} p_{z^{1.29}}$ \\
\hline
\end{tabular}

Table 3 contains the results of the IQA analysis of type-A $\mathrm{CE}_{2} \mathrm{Li}_{2}(\mathrm{E}=\mathrm{Si}-\mathrm{Pb})$, which further support the characterized bonding scheme. The substantial exchange-correlation energy component $(-212.4--181.4 \mathrm{kcal} / \mathrm{mol})$ of C-E relative to the marginal C-M interactions $(-18.4--16.7 \mathrm{kcal} / \mathrm{mol})$ confirm the polar covalent (C-E) and electrostatic (C-M) bonding nature, respectively. Note that IQA predicts that $\mathrm{C}-\mathrm{M}$ interactions are attractive (mainly Coulombic) in total agreement with the partial charge analysis. Additionally, the C-E, C-M, and E-Li delocalization indices ( $\delta$ ), an estimation of the bond order within the IQA methodology, support that $\mathrm{C}-\mathrm{E}$ is a double bond $(\delta=1.6-1.8)$. In contrast, $\mathrm{C}-\mathrm{M}(\delta=0.1-0.2)$ and $\mathrm{E}-\mathrm{M}(\delta=0.1)$ are dominated by electrostatic interactions, in total agreement with the WBI analysis. Similar IQA trends were obtained for type-B isomers. The results are included in Table S2.

The $\mathbf{B}^{\text {ind }}$ analysis of the $\mathbf{A}$ and $\mathbf{B}$ isomers of $\mathrm{CSi}_{2} \mathrm{Li}_{2}$ shows a diatropic current (in blue) flowing around the Si-C-Si framework and inducing a shielding cone in that region. Concomitantly, the lithium atoms are deshielded (characterized by positive $B^{\text {ind }} z$ values) due to a local paratropic current (in red) in the vicinity of their nuclei. Note that the $\sigma$-delocalization in the Si-C-Si framework is the dominant one in both structures (Fig. 3). The diatropic current depicted in $\mathrm{CSi}_{2} \mathrm{Li}_{2}(\mathbf{A})$ is interrupted in the corresponding type-A systems with heavier $\mathrm{E}$ atoms, decreasing the $\sigma$-shielding (Fig. S12).
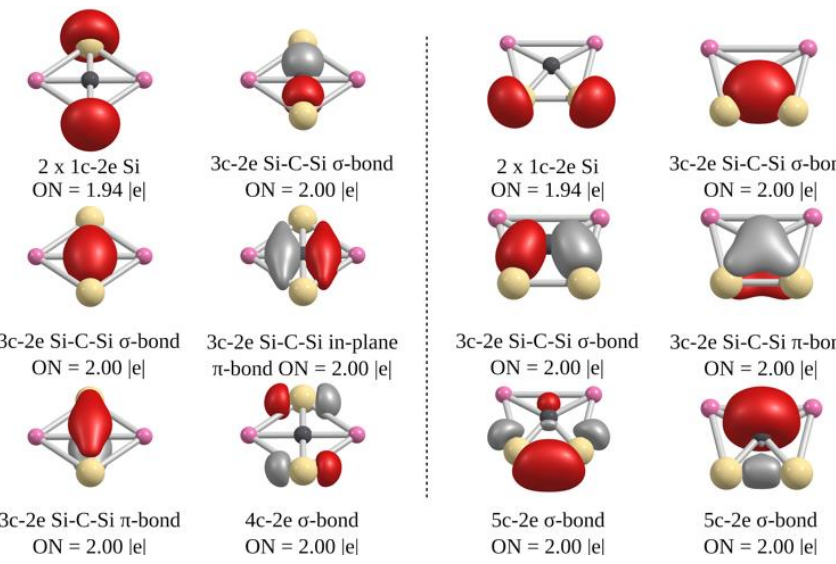

3c-2e Si-C-Si o-bond $\mathrm{ON}=2.00|\mathrm{e}|$

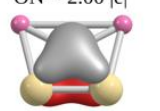

3c-2e Si-C-Si o-bond 3c-2e Si-C-Si $\pi$-bond $\begin{array}{ll}\mathrm{ON}=2.00|\mathrm{e}| & \mathrm{ON}=2.00|\mathrm{e}|\end{array}$

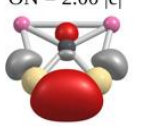

5c-2e o-bond $\mathrm{ON}=2.00$ le

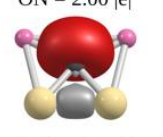

5c-2e o-bond $\mathrm{ON}=2.00$ le

Fig. 2. AdNDP analysis of type-A (left) and B (right) ptCs of CSi2Li2. ON stands for occupation number.
Remarkably, the bending of the Si-C-Si fragment induces a "single" Si-Si bond ( $\mathrm{WBI}=1.14)$, leading to the electron delocalization in the Si-C-Si triangle, so this moiety can be considered aromatic. This diatropic pathway around $\mathrm{E}=\mathrm{C}=\mathrm{E}$ was also found in the remaining $\mathbf{B}$ isomers (see Fig. S13).

So, if aromaticity in the $E=C=E$ ring leads to global minima adopting the type-B form, why is it not the most stable isomer in the sodium cases? To answer this question, we compute the $\mathrm{E}=\mathrm{C}=\mathrm{E}$ dianions corresponding to the angular and linear fragments. Contrary to what is expected, the linear motif is lower in energy than the angular one by $6.13,6.93,5.47$, and $3.41 \mathrm{kcal} / \mathrm{mol}$ as moving from $\mathrm{Si}$ to $\mathrm{Pb}$. In other words, although aromaticity could stabilize the type-B structures, an important factor is the repulsion between cations. This explains why, in $\mathrm{Na}$-clusters, the most favorable structure is the one with the most distant cations (type-A).

Table 3. Energy components ( $\mathrm{kcal} / \mathrm{mol}$ ) of IQA for type-A $\mathrm{CE}_{2} \mathrm{Li}_{2}(\mathrm{E}=\mathrm{Si}, \mathrm{Ge}, \mathrm{Sn}$, and $\mathrm{Pb})$. $V_{I Q A}^{\text {int }}, V_{C}^{\text {int }}$, and $V_{X C}^{\text {int }}$ are interatomic IQA interaction energy, coulombic and exchangecorrelation energy components, respectively. The delocalization indices are represented by $\delta$. Values between parentheses correspond to Na-clusters.

\begin{tabular}{ccccc}
\hline & $\mathrm{CSi}_{2} \mathrm{Li}_{2}$ & $\mathrm{CGe}_{2} \mathrm{Li}_{2}$ & $\mathrm{CSn}_{2} \mathrm{Li}_{2}$ & $\mathrm{CPb}_{2} \mathrm{Li}_{2}$ \\
\hline$V_{I Q A}^{I n t}(C-E)$ & $-773.4(-683.0)$ & $-386.5(-346.8)$ & $-310.4(-277.7)$ & $-251.2(-226.3)$ \\
$V_{C}^{I n t}(C-E)$ & $-587.2(-501.6)$ & $-174.0(-140.1)$ & $-117.3(-90.2)$ & $-63.5(-44.5)$ \\
$V_{X C}^{I n t}(C-E)$ & $-186.2(-181.4)$ & $-212.4(-206.7)$ & $-193.2(-187.5)$ & $-187.7(-181.9)$ \\
$V_{I Q A}^{I n t}(C-M)$ & $-408.8(-308.78)$ & $-289.1(-213.3)$ & $-270.5(-194.3)$ & $-247.0(-174.1)$ \\
$V_{C}^{I n t}(C-M)$ & $-390.4(-290.6)$ & $-272.4(-196.6)$ & $-253.4(-177.3)$ & $-229.6(-156.5)$ \\
$V_{X C}^{I n t}(C-M)$ & $-18.4(-18.2)$ & $-16.7(-16.7)$ & $-17.1(-17.0)$ & $-17.4(-17.6)$ \\
$V_{I Q A}^{I n t}(E-M)$ & $82.9(52.4)$ & $23.8(6.2)$ & $15.7(-0.2)$ & $3.6(-9.3)$ \\
$V_{C}^{I n t}(E-M)$ & $87.5(58.5)$ & $29.2(13.2)$ & $20.8(6.9)$ & $8.7(-2.1)$ \\
$V_{X C}^{I n t}(E-M)$ & $-4.5(-6.1)$ & $-5.4(-7.0)$ & $-5.1(-7.1)$ & $-5.1(-7.2)$ \\
$\delta(C-E)$ & $1.6(1.6)$ & $1.8(1.7)$ & $1.7(1.7)$ & $1.7(1.7)$ \\
$\delta(C-M)$ & $0.2(0.2)$ & $0.1(0.2)$ & $0.1(0.2)$ & $0.1(0.2)$ \\
$\delta(E-M)$ & $0.1(0.1)$ & $0.1(0.1)$ & $0.1(0.1)$ & $0.1(0.1)$ \\
\hline
\end{tabular}




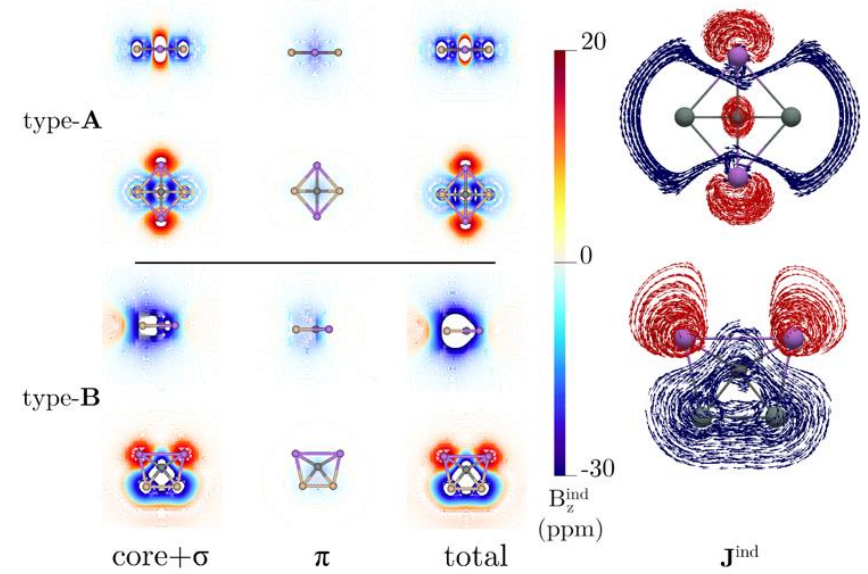

Fig. 3. The isolines of the orbital contributions to $B^{\text {ind }}{ }_{2}$ for isomers $\mathbf{A}$ and $\mathbf{B}$ of $\mathrm{CSi}_{2} \mathrm{Li}_{2}$ in the left panel. $J^{\text {ind }}$ vector maps in the right panel. The diatropic and paratropic currents are shown in blue and red, respectively. All the computations were performed at the TPSS/TZP-DKH level.

In summary, seven systems of the $\mathrm{CE}_{2} \mathrm{M}_{2}(\mathrm{E}=\mathrm{Si}-\mathrm{Pb}, \mathrm{M}=\mathrm{Li}$, and $\mathrm{Na}$ ) combinations were identified as global minima with a ptC. The series was proposed according to the $\pi$-localization strategy, in which the central carbon forms a double bond with each atom of group 14. As a result, two different $E=C=E$ motifs arise, a linear one with an unusual $s p$ hybridization of the carbon (A) and a bent one with an $s p^{2}$ hybridization (B), which according to the magnetic response, have a $E=C=E$ fragment with $\sigma$-aromaticity. In contrast, the interactions of the center with the alkali-metals are predominantly electrostatic. The formation of multiple bonds enhances the stabilization of these systems, which contrasts with the traditional approach of using $\pi$-acceptor/ $\sigma$-donor ligands.

This work was funded by the National Natural Science Foundation of China (No. 11922405, 11874178, 91961204), and supported by Beijing National Laboratory for Molecular Sciences (BNLMS201910). The work in Mexico is supported by Grant SEP-Cinvestav-2018-57. J. B. and M. O.-I. thank Conacyt for their PhD fellowships.

There are no conflicts to declare.

\section{Notes and references}

1 J. H. van't Hoff, Arch. Neerl. Sci. Exactes Nat., 1874, 9, 445454.

2 J. A. LeBel, Bull. Soc. Chim. Fr., 1874, 22, 337-347.

3 K. B. Wiberg, Chem. Rev., 1989, 89, 975-983.

4 P. E. Eaton and G. H. Temme, J. Am. Chem. Soc., 1973, 95, 7508-7510.

5 R. M. Minyaev, T. N. Gribanova, V. I. Minkin, A. G. Starikov and R. Hoffmann, J. Org. Chem., 2005, 70, 6693-6704.

6 J. P. Kenny, K.M. Krueger, J. C. Rienstra-Kiracofe and H. F. Schaefer III, J. Phys. Chem. A 2001, 105, 7745-7750.

7 D. R. Rasmussen and L. Radom, Chem. Eur. J., 2000, 6, 24702483.

8 J. S. Bradley, G. B. Ansell, M. E. Leonowicz and E. W. Hill, J. Am. Chem. Soc., 1981, 103, 4968-4970.
9 A. Gunale, H. Pritzkow, Siebert, D. Steiner and A. Berndt, Angew. Chem. Int. Ed. Engl. 1995, 34, 1111-1113.

10 U. Radius, S. J. Silverio, R. Hoffmann and R. Gleiter, Organometallics, 1996, 15, 3737-3745.

11 R. Hoffmann, R. W. Alder and C. F. Wilcox, J. Am. Chem. Soc., 1970, 92, 4492-4493.

12 H. J. Monkhorst, Chem. Commun., 1968, 1111-1112

13 P. v. R. Schleyer and A. Boldyrev, J. Chem. Soc., Chem Commun., 1991, 21, 1536-1538.

14 A. I. Boldyrev and J. Simons, J. Am. Chem. Soc., 1998, 120, 7967-7972.

15 G. Erker, Chem. Soc. Rev., 1999, 28, 307-314.

16 W. Siebert and A. Gunale, Chem. Soc. Rev., 1999, 28, 367371.

17 R. Keese, Chem. Rev., 2006, 106, 4787-4808.

18 G. Merino, M. A. Mendez-Rojas, A. Vela and T. Heine, J. Comput. Chem., 2007, 28, 362-372.

19 L. M. Yang, E. Ganz, Z. F. Chen, Z. X. Wang and P. V. Schleyer, Angew. Chem., Int. Edit., 2015, 54, 9468-9501.

20 J. C. Guo, L. Y. Feng and H. J. Zhai, Phys. Chem. Chem. Phys., 2018, 20, 6299-6306.

21 J. C. Guo, L. Y. Feng, C. Dong and H. J. Zhai, J. Phys. Chem. A, 2018, 122, 8370-8376.

22 G. Merino, M. A. Mendez-Rojas and A. Vela, J. Am. Chem. Soc., 2003, 125, 6026-6027.

23 S.-D. Li, G.-M. Ren, C.-Q. Miao and Z.-H. Jin, Angew. Chem., Int. Ed., 2004, 43, 1371-1373.

24 G. Merino, M. A. Mendez-Rojas, H. I. Beltraan, C. Corminboeuf, T. Heine and A. Vela, J. Am. Chem. Soc., 2004, 126, 16160-16169.

25 P. D. Pancharatna, M. A. Mendez-Rojas, G. Merino, A. Vela and R. Hoffmann, J. Am. Chem. Soc., 2004, 126, 1530915315.

26 H. B. Xie and Y. H. Ding, J. Chem. Phys., 2007, 126.

27 Y. B. Wu, J. L. Jiang, H. Li, Z. F. Chen and Z. X. Wang, Phys. Chem. Chem. Phys., 2010, 12, 58-61.

28 Z. H. Cui, M. Contreras, Y. H. Ding and G. Merino, J. Am. Chem. Soc., 2011, 133, 13228-13231.

29 Y. B. Wu, Z. X. Li, X. H. Pu and Z. X. Wang, J. Phys. Chem. C, 2011, 115, 13187-13192.

30 Y. B. Wu, Z. X. Li, X. H. Pu and Z. X. Wang, Comput. Theor. Chem., 2012, 992, 78-83.

31 M. Contreras, S. Pan, M. Orozco-Ic, J. L. Cabellos and G. Merino, Chem. -Eur. J., 2017, 23, 11430-11436.

32 O. Yanez, A. Vasquez-Espinal, R. Pino-Rios, F. Ferraro, S. Pan, E. Osorio, G. Merino and W. Tiznado, Chem. Commun., 2017, 53, 12112-12115.

33 H. F. Zheng, J. Xu and Y. H. Ding, Phys. Chem. Chem. Phys., 2020, 22, 3975-3982.

34 Z. H. Cui, C. B. Shao, S. M. Gao and Y. H. Ding, Phys. Chem. Chem. Phys., 2010, 12, 13637-13645.

35 Z. H. Cui, Y. H. Ding, J. L. Cabellos, E. Osorio, R. Islas, A. Restrepo and G. Merino, Phys. Chem. Chem. Phys., 2015, 17 8769-8775

36 J. Y. Guo, H. Y. Chai, Q. Duan, J. M. Qin, X. D. Shen, D. Y. Jiang, J. H. Hou, B. Yan, Z. R. Li, F. L. Gu and Q. S. Li, Phys. Chem. Chem. Phys., 2016, 18, 4589-4593.

37 Z. H. Cui, V. Vassilev-Galindo, J. L. Cabellos, E. Osorio, M. Orozco, S. Pan, Y. H. Ding and G. Merino, Chem. Commun. 2017, 53, 138-141.

38 M.-h. Wang, X. Dong, Z.-h. Cui, M. Orozco-lc, Y.-h. Ding, J. Barroso and G. Merino, Chem. Commun., 2020, 56, 13772 13775 .

39 L. Leyva-Parra, L. Diego, O. Yañez, D. Inostroza, J. Barroso, A Vásquez-Espinal, G. Merino and W. Tiznado, Angew. Chem. Int. Edit., in press.

40 P. Pyykko, J. Phys. Chem. A, 2015, 119, 2326-2337. 